\section{Drug Design I-Small Molecule Based}

MS05.01.01 STRUCTURAL ANALYSIS OFRAPAMYCIN'S ROLE IN BINDING FKBP12 AND FRAP. Jon Clardy*, Jungwon Choi*, Jie Chen $\%$, and Stuart L. Schreiber:, *Department of Chemistry, Cornell University, Ithaca, NY 14853-1301. $\$$ Howard Hughes Medical Institute, Department of Chemistry, Harvard University, Cambridge, MA 02138, USA

The immunosuppressive drug rapamycin works by binding together two proteins: the FK506 binding protein (FKBP12) and the FKBP-rapanycin associated protein (FRAP). The crystal structure of the immunosuppressive agent rapanycin complexed with human FK506 binding protein (FKBP12) and the FKBP12rapamycin binding domain (FRB) of FKBP12-rapamycin associated protein (FRAP), reveals two proteins bound together through rapamycin's ability to simultaneously occupy two different hydrophobic binding pockets. FKBPI2 has a hydrophobic binding pocket between a large $\beta$-sheet and a short amphipathic $\alpha$-helix. FRB is a four-helix bundle, and the crossing region between two antiparallel helices forms the rapanycin binding pocket. The structure shows extensive interactions between rapamycin and both proteins but only modest protein-protein interactions. The structure also provides, among other things, an understanding of the critical nature of the serine 2,035 residue, and the first structural information about the growing family of proteins related to the ataxia telangicetasia mutant (ATM) gene product.

Crystals of the triple complex form in space group $\mathrm{P} 2_{1} 2_{1} 2_{1}$ with $\mathrm{a}=44.63, \mathrm{~b}=52.14$, and $\mathrm{c}=102.53 \AA$ and one FKBP12rapanycin-FRB complex in the asymmetric unit. Data were collected to $2.7 \AA$ with a rotating anode source. A phasing model was found using MR (FKBP12) and SIRAS ( $\mathrm{HgCl}_{2}$ derivative). The current model has $\mathrm{R}=19.3 \%$ (Rfree $=29.9 \%$ ) for the 6206 reflections between 8 and $2.7 \AA$ and good geometry (r.m.s. deviations of $0.008 \AA$ for bond lengths and $1.48^{\circ}$ for bond angles) for a model with 1639 protein atoms, 65 rapamycin atoms, and 23 solvent atoms-not counting hydrogens.

MS05.01.02 THE RELEVANCE OF THE CRYSTAL STRUCTURE OF HUMAN ENDOTHELIN TO G-PROTEIN COUPLED RECEPTOR BINDING. B.A. Wallace and R.W. Janes, Dept. of Crystallography, Birkbeck College, University of London, London, England

The recently solved (Nature Struct. Biol. 1:311-319) crystal structure of human endothelin-1 the most potent naturally-occurring vasoconstrictor, has revealed structural features of the polypeptide that differ considerably from those found in the reported structures of endothelin as determined by NMR spectroscopy (Prot. Sci. 4:7583). The most significant differences are in those regions important for receptor binding and specificity, namely the central loop and the C-terminal helix. Comparisons of the crystal structure with the structures of chemically-unrelated but very potent small molecule antagonists show (FEBS Lett. 374:379-383) a strong correspondence in their 3-dimensional structures. These, and correlations between its structural features and binding and activity data for a larse number of naturallyoccurring and synthetic mutants strongly support the crystal structure as being biologically relevant with respect to binding to G-Protein coupled receptors. (Supported by a grant from the British Heart Foundation).
MS05.01.03 CONSTRAINED LINEAR OPIOHD PEPTIDES. Jeffrey R. Deschamps, Clifford George, Christi Moore, Robert Cudney*, and Judith L. Flippen-Anderson, Laboratory for the Structure of Matter, Naval Research Laboratory, Washington, DC, 20375 and :Hampton Research, Laguna Hills, CA.

A new class of potent site selective opioid peptides has been developed which consist entirely of aromatic amino acids and contain a conformationally restricted phenylalanine residue (i.e. tetrahydroisoquinoline-3-carboxylic acid, Tic) in position 21. More recent work on these short linear peptides has shown that aromaticity of residues 3 and 4 is not required for high binding affinity 2 . Members of this family that contain a L-Tic residue in position 2 are $\delta$ selective antagonists and those that contain a D-Tic at position 2 are agonists with some preference for $\mu$ receptors. Even the dipeptide (Tyr-Tic$\mathrm{NH}_{2}$ ) has been shown to possess substantial opioid activity3. We have determined the structures of several members of this new class of opioid peptides. A comparison of these structures reveals a relationship between the positions of the $\mathrm{Tyr}^{1}$ and $\mathrm{Tic}^{2}$ side chains and the activity of the peptide ligand. In TIPP (Tyr-Tic-Phe-Phe), a highly $\delta$ selective antagonist, the distance between these side chains is $5.9 \AA$; in the agonist D-TIPP (Tyr-D-Tic-Phe-Phe) the distance between these side chains is approximately $6.5 \AA$. Shorter ring-ring distances are associated with a conformation of enkephalin thought to represent that of an antagonist, while longer ring-ring distances are associated with a conformation which is thought to be required for agonist activity.

1. P.W. Schiller, et al. (1992) Proc. Nat. Acad. Sci, 89, 11871-11875.

2. H.I. Mosberg, et al. (1994) Lett. Pep. Sci., 1, 69-72.

3. P.A. Temussi, et al. (1994) Biochem Bioplys Res. Comm., 198, $933-939$.

MS05.01.04 CONFORMATIONAL CHANGES OF SMALL MOLECULES BINDING TO PROTEINS - COMPARISON OF CSD AND PDB STRUCTURES. Marc C. Nicklaus, Shaomeng Wang, John S. Driscoll, and George W. A. Milne; Laboratory of Medicinal Chemistry, Division of Basic Sciences, National Cancer Institute, National Institutes of Health, Bldg. 37, Rm. 5B29, 37 Convent Drive, Bethesda, MD 20892-4255, USA.

Flexible molecules change their conformation upon binding to a protein. This was shown by the analysis of small molecules whose structures have been determined by $\mathrm{x}$-ray crystallography of both the pure compound and the compound bound to a protein. Thirtythree compounds present both in the Cambridge Structural Database and the Brookhaven Protein Data Bank were analyzed, and both were compared with the global energy minimum conformation in vacuum, computed by molecular mechanics force fields calculations. It was found that the conformation bound to the protein differs from that in the crystal structure and also from that of the global energy minimum, and the degree of deformation depends upon the number of freely rotatable bonds in the molecule. Four rotatable bonds appeared to be a limit beyond which no great similarities can generally be expected between the crystal, proteinbound, and vacuum conformations. Analysis of the conformational energies of the flexible molecules showed that, for most of those compounds, both the crystal and the protein-bound conformations are energetically well above the vacuum global minimum, and, in many cases, not even in any local energy minimum. Semiempirical calculations performed for a select number of structures, using both the AM1 and PM3 hamiltonians, confirmed these results. The number of ligand atoms forming hydrogen bonds was found to be correlated with the energy available for deformation of the ligand. The accuracy of the determination of the conformation of protein-bound small molecules is discussed in the context of the resolution of the crystallographic structures. These findings are discussed as to their impact upon contemporary methods of drug design. 